\title{
Brinquedo terapêutico reduz a ansiedade em procedimentos odontológicos? Estudo clínico randomizado
}

\author{
Does therapeutic play reduce anxiety in dental procedures? Randomized clinical study \\ ¿El juego terapéutico reduce la ansiedad en los procedimientos dentales? Estudio clínico \\ aleatorizado
}

Maria Tereza Freire Carvalho ${ }^{1,3 *}$, Sérgio Luiz Pinheiro², Giselle Rodrigues de Sant'Anna ${ }^{3}$, Danilo Antonio Duarte ${ }^{3}$.

\section{RESUMO}

Objetivo: Avaliar a influência do brinquedo terapêutico sobre o grau de ansiedade produzida durante procedimento odontológico não invasivo. Métodos: Foram avaliadas 212 crianças, ambos os sexos, na faixa de 24 a 36 meses. Pesquisa realizada em duas creches municipais de uma cidade do Maranhão, após aprovação do CEP-CAAE 08426519.7.0000.5374. Estabeleceu-se Grupo Experimental - Brinquedo Terapêutico (BT), $n=107$, com uso BT e o Grupo Controle (CTR), $n=105$, sem uso do BT. Utilizou-se medidas de batimento cardíaco e saturação de Oxigênio, bem como Teste VPT, FIS e FIS (Turma da Mônica), tomadas antes e após o procedimento, em ambos os grupos. Empregou-se teste t de Student para comparação entre variáveis numéricas, Mann Whitney para escalas de ansiedade e Spearman para correlação. Resultados: Houve redução do grau de ansiedade pelo Venham Picture Test (VPT) e Escalas Faciais (FIS) adaptada e Turma da Mônica após uso do BT, bem como redução da frequência cardíaca. Observou-se correlação entre batimentos por minuto e escalas de ansiedade e entre elas $(p=0,01)$, após procedimento. Conclusão: $A$ aplicação do BT promoveu redução do nível de ansiedade, tornando as crianças mais receptivas ao procedimento odontológico. Revelou-se, ainda, um método positivo e apropriado para estimular seu uso na rotina clínica odontopediátrica.

Palavras-chave: Jogos e brinquedo, Escala de ansiedade, Comportamento infantil.

\begin{abstract}
Objective: To evaluate the influence of therapeutic toys on the degree of anxiety produced during non-invasive dental procedures. Methods: 212 children, both genders, aged 24 to 36 months, were evaluated. Research carried out in two municipal daycare centers in a city in Maranhão, after approval by CEP-CAAE 08426519.7.0000.5374. The Therapeutic Toy Experimental Group (BT), $n=107$, with BT use and the Control Group (CTR), $n=105$, without BT were established. Heart rate and oxygen saturation measurements were used, as well as VPT, FIS and FIS (Turma da Mônica) test, taken before and after the procedure, in both groups. Student's t test was used to compare numerical variables, Mann Whitney for anxiety scales and Spearman for correlation. Results: There was a reduction in the degree of anxiety by the Venham Picture Test (VPT) and adapted Facial Scales (FIS) and Turma da Mônica after using BT, as well as a reduction in heart rate. There was a correlation between beats per minute and anxiety scales and between them $(p=0.01)$ after the procedure. Conclusion: The application of BT promoted a reduction in the level of anxiety, making children more receptive to the dental procedure. It also proved to be a positive and appropriate method to encourage its use in the pediatric clinical routine.
\end{abstract}

Key words: Games and toys, Anxiety scale, Child behavior.

\section{RESUMEN}

Objetivo: Evaluar la influencia de los juguetes terapéuticos en el grado de ansiedad que se produce durante los procedimientos dentales no invasivos. Métodos: se evaluaron 212 niños de ambos sexos, de 24 a 36 meses de edad. Investigación realizada en dos guarderías municipales de una ciudad de Maranhão, previa

\footnotetext{
1 Universidade Estadual do Maranhão (UEMA), Bacabal - MA. *E-mail: terezafc@hotmail.com

2 Faculdade de Odontologia (PUC), Campinas - SP.

${ }^{3}$ Centro de Pesquisas São Leopoldo Mandic, Campinas - SP.
} 
aprobación CEP-CAAE 08426519.7.0000.5374. Se establecieron el Grupo Experimental de Juguete Terapéutico (BT), $n=107$, con uso de BT y el Grupo Control (CTR), $n=105$, sin BT. Se utilizaron medidas de frecuencia cardíaca y saturación de oxígeno, así como pruebas de VPT, FIS y FIS (Turma da Mônica), realizadas antes y después del procedimiento, en ambos grupos. Se utilizó la prueba t de Student para comparar variables numéricas, Mann Whitney para escalas de ansiedad y Spearman para correlación. Resultados: Hubo una reducción en el grado de ansiedad por el Venham Picture Test (VPT) y las Escalas faciales adaptadas (FIS) y Turma da Mônica después de usar BT, así como una reducción en la frecuencia cardíaca. Hubo una correlación entre los latidos por minuto y las escalas de ansiedad y entre ellos $(p=0.01)$ después del procedimiento. Conclusión: La aplicación de BT promovió una reducción del nivel de ansiedad, haciendo que los niños fueran más receptivos al procedimiento dental. También resultó ser un método positivo y apropiado para incentivar su uso en la rutina clínica pediátrica.

Palabras clave: Juegos y juguetes, Escala de ansiedad, Comportamiento infantil.

\section{INTRODUÇÃO}

Procedimentos odontológicos representam uma circunstância que difere de todas as vivenciadas pela criança. Nesse contexto, está circundada por um ambiente impessoal, relacionando com pessoas desconhecidas sendo submetidas a procedimentos com potencial de provocar desconfortos (BARROSO MCCS, et al., 2020). Por sua vez, o desconforto produz, especialmente em crianças de tenra idade, elevado grau de ansiedade e medo, motivos preponderantes na recusa ou não adesão a eventuais e necessárias intervenções odontológicas, sejam elas invasivas ou não (RIBEIRO WA, et al, 2020; GOÉS FGB, et al, 2019). A ansiedade é compreendida como uma resposta a situações nas quais a origem da ameaça ao indivíduo não é objetivamente real ou presente (GÓES MP, et al., 2010).

Dentre vários aspectos que motivam o binômio paciente/profissional da saúde na redução da ansiedade, ressalta-se o "brincar e o brinquedo", como expressivas funções facilitadoras e mediadoras nas intervenções clínicas (RIBEIRO WA, et al., 2020).

O brincar é uma ação capaz de reequilibrar a criança e suas emoções, bem como concentrar sua atenção em um determinado procedimento. O brinquedo, considerado terapêutico (BT), conceitua-se como um brinquedo estruturado que promove o alívio da ansiedade causada por experiências atípicas da idade e facilita o entendimento da criança na vivência de procedimentos invasivos e dolorosos, incentivando-as a enfrentar de forma positiva o momento vivenciado (CARVALHO FS, et al., 2015).

A ansiedade provocada em procedimentos odontológicos não é de fácil aferição por incorporar medidas subjetivas, influência do núcleo familiar e do profissional além do tipo de procedimento. A despeito desses fatores, estratégias são comumente utilizadas para tal como: Venham Picture Test (VPT), Escalas de Faces adaptadas e a FIS Turma da Monica (FIS adaptada), dentre outras. Sinais vitais como frequência cardíaca e saturação de oxigênio também podem refletir o grau de ansiedade durante atividades clínicas (TSHISWAKA SK e PINHEIRO SL, 2020; OZKALAYCI O, et al., 2016).

Dessa forma, objetivou-se avaliar a influência do BT sobre o grau de ansiedade, em crianças de 24 a 36 meses de idade, produzida durante procedimento odontológico não invasivo.

\section{MÉTODOS}

A pesquisa foi realizada em uma cidade do estado do Maranhão - Brasil. O desenho do estudo foi experimental, descritivo, enfoque qualitativo, em crianças da educação infantil, realizado período de março/2019 a dezembro/2019, após aprovação pelo Comitê de Ética em Pesquisa (CEP) do Centro de Pesquisas São Leopoldo Mandic, CAAE 08426519.7.0000.5374, atendendo às exigências éticas e científicas referente a Normas de Pesquisa envolvendo Seres Humanos.

\section{Cálculo e seleção da amostra}

Foi calculada por meio do Programa EPIINFO, utilizando STAT CALC, a partir de uma população de 496 crianças, matriculadas em creches públicas de uma cidade do Maranhão, considerando os seguintes critérios: 
frequência esperada de 50\%; margem de erro 5\%; nível de significância de 95\%. Amostragem redundou em 217 crianças e após aplicado critérios de exclusão: a ausência da criança no momento da pesquisa, crianças com limitações cognitivas, alterações sistêmicas ou síndromes, crianças com experiências odontológicas anteriores (acima de duas consultas odontológicas) internações médicas; associada a perdas ocorridas no estudo e em conformidade com os critérios de inclusão: faixa etária 24 a 36 meses, matriculadas nas creches organizadas em regime parcial, cujos responsáveis assinaram Termo de Consentimento Livre e Esclarecido (TCLE) e aceitem participar da execução da pesquisa, totalizou-se 212 crianças.

\section{Caracterização do ambiente da pesquisa}

O município possui 48 creches públicas cadastradas no Programa Saúde na Escola (PSE) e dessas, duas foram aleatoriamente selecionadas, ambas situadas em zona urbana, possuindo características físicas estruturais e pedagógicas similares. A randomização das creches foi realizada através de sorteio, assim como, dos grupos dos alunos integrantes da pesquisa: duas creches, dessas duas creches sorteamos duas turmas da educação infantil e posteriormente, os grupos de alunos.

\section{Instrumentos e procedimentos da Metodologia}

A implementação da metodologia desenvolvida atendeu às recomendações previstas no Consolidated Standards of Reporting Trials (CONSORT) (DIMAIRO M, et al., 2020). Direcionado a pesquisa clínica composto por uma lista de checagem (check list) de 22 itens. As recomendações do CONSORT destinam-se a aperfeiçoar o relato de qualquer exame clínico randomizado, levando o leitor a compreender melhor a condução do estudo.

\section{Estudo Piloto}

Realizou-se um estudo piloto, amostra de 5 crianças, que não participaram do projeto principal. $O$ objetivo do estudo piloto foi calibrar os examinadores e avaliar os instrumentos da pesquisa. Kappa 0,9 entre os examinadores referente a aplicação do Teste e Escalas, e Kappa 1,0 intra examinador que aferiu os batimentos cardíacos e saturação de oxigênio.

\section{Dados não clínicos}

Para coleta de dados não clínicos foi elaborado um questionário, contendo informações sócio demográficas e história médica-odontológica adaptada (CÉ L, 2002) preenchido pelo responsável da criança.

Para avaliar o grau de ansiedade da população estudada valeu-se do Venham Picture Test (VPT) (VENHAM LL, et al., 1980), considerado o instrumento indicado para pré-escolares, caracterizado como teste projetivo com autoanálise a partir de desenho de figuras humanas (SOARES JP, et al., 2020). Utilizou-se também a Facial Image Scale (FIS) (RANK RCIC e SILVA DRP, 2002), constituída por expressões faciais e a FIS - Turma da Monica (CLARO MT, 1993) ambos, modelos adequados para avaliar ansiedade e de alta confiabilidade (MELO RB, et al., 2015).

\section{Dados Clínicos}

Relativo aos parâmetros biológicos recorreu-se aos sinais vitais: frequência cardíaca e saturação do oxigênio. Descrito por Ghadimi S, et al. (2018) aferida pelo estetoscópio ou oxímetro. Optou-se pelo oxímetro pediátrico de dedo (G Tech Oled Graph) por oferecer: medida direta de excitação fisiológica, leitura regular e imediata; utilizando parâmetro normal 100 bpm; seu aumento é atribuído ao estresse (OZKALAY CI, et al., 2016; MARWAH N, et al., 2005). As crianças sentarem-se relaxadas e, foi posicionado o oxímetro no dedo indicador, aguardando 5 minutos para registrar o valor.

Quanto ao procedimento odontológico, elegeu-se a técnica Tratamento Restaurador Atraumático (ART), estrategicamente utilizada por representar uma filosofia de mínima intervenção, menor desconforto ao paciente, sem necessidade de anestesia e de instrumentos rotatórios. Consistia na remoção seletiva da dentina cariada, com instrumentos manuais, posterior restauração da cavidade com cimento de ionômero de vidro, proteção com vaselina ou verniz e seleção de dentes envolvidos preconizado pela técnica ART (FRENCKEN JE, 2017). Instrumentais e materiais utilizados: Máscara, luva, gorro, avental, curetas, algodão, 
gaze, cimento restaurador de ionômero de vidro (Riva Self Cure-SDI) e vaselina. A aferição foi realizada antes e após o procedimento.

\section{Composição dos grupos e fluxo dos procedimentos (Figura 1)}

Figura 1 - Fluxograma demonstrando a composição dos grupos e o fluxo dos procedimentos podendo ser observada as etapas do desenvolvimento da pesquisa.

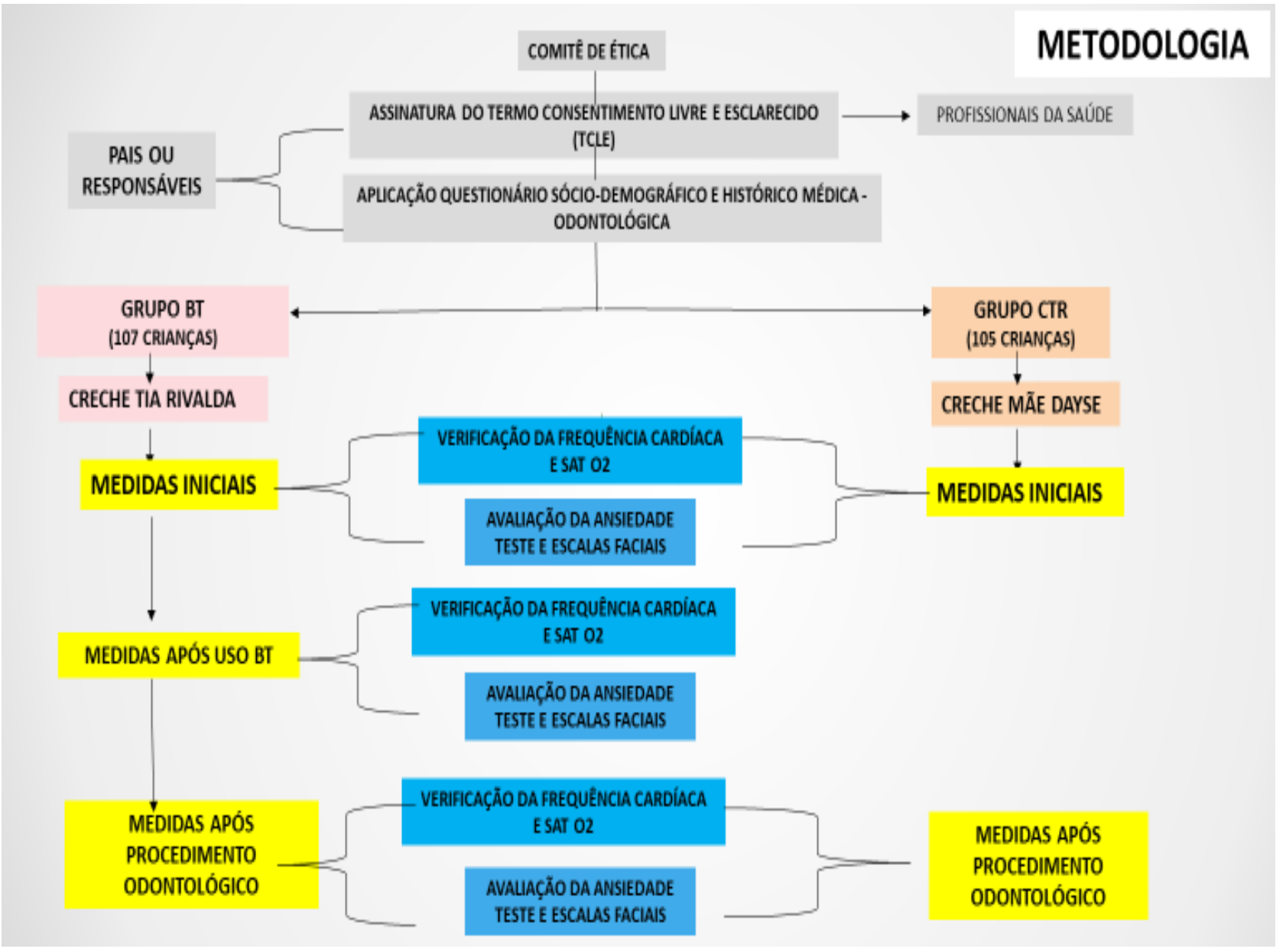

Fonte: Carvalho MTF, et al., 2019.

Utilizou-se: iluminação natural, profissionais com proteção individuais, atendimento nas creches, examinadas posição joelho-a-joelho. O brinquedo terapêutico (BT), o Play Doh Dentista foi apresentado ao grupo intervencional (BT) utilizando a técnica mostrar-falar-fazer antes do procedimento odontológico (ART) (ZHU M, et al., 2020). As crianças exercitaram no BT tarefa semelhante ao procedimento. Tempo médio com o BT foi 8 a 10 minutos e de cada sessão 25 a 30 minutos. No grupo controle realizou-se acolhimento sem utilização da técnica; antes e após a ART repetia-se as medições.

\section{Análise estatística}

Os dados inicialmente inseridos em três planilhas Excel®, consolidados em dois modelos estatísticos, onde, para análise exploratória dos dados foram usadas as técnicas gráficas: Boxplots - Representação gráfica das cinco medidas resumo de um conjunto de dados; Diagramas Aluviais - Representação de tabelas de variáveis categóricas eixos paralelos verticais representam as variáveis e os seus níveis por blocos em cada um desses eixos; Diagramas de Coordenadas Paralelas - Representação de variáveis numérica em eixos paralelos onde os valores para os casos são conectado via linhas para cada variável permitindo ver suas medidas em "feixes" de valores para toda a amostra; Gráficos de Contingência - Também denominados gráficos de produto na literatura, apresentam um diagrama similar a um diagrama de dispersão com 0 
tamanho dos símbolos proporcional às frequências dos cruzamentos da tabela de contingência representada.

$\mathrm{Na}$ análise inferencial foram efetuados os testes estatísticos para responder às questões levantadas na pesquisa. Para comparação entre as variáveis numéricas empregou-se o teste t de Student com correção para variâncias distintas em cada perna da amostra; para comparações das medidas das escalas de ansiedade empregou-se teste Mann Whitney para grupos independentes; para verificação da "equivalência" entre as várias escalas de ansiedade empregou-se a correlação de postos de Spearman, apresentados na matriz de diagramas de dispersão. Os dados foram analisados através do software estatístico $R$ for Windows versão 3.6.2.

\section{RESULTADOS}

A Tabela 1 identifica dados sociodemográficos da população estudada incluindo atributos como: idade, sexo e renda. Exibe as medidas iniciais do grupo de intervenção (brinquedo terapêutico) antes e após a aplicação do BT e após o procedimento odontológico (ART) e as medidas iniciais do grupo controle (CTR) e após o procedimento odontológico. Observa-se que a diferença de idades das crianças entre os dois grupos foi de um mês e as proporções dos sexos se mantiveram em relação à proporção do total. Em relação à renda o valor reportado foi de 1 salário-mínimo (1SM) com proporções similares a amostra total. Em referência às medidas de ansiedade iniciais percebe-se uma diferença de $5 \mathrm{bpm}$, entre as médias dos dois grupos. Medidas tomadas antes de qualquer intervenção ou exposição das crianças.

Tabela 1 - Referente a idade, sexo e renda e as medidas iniciais antes aplicação do BT e do procedimento não invasivo (ART) e após.

\begin{tabular}{|c|c|c|c|c|c|}
\hline Variáveis & $\begin{array}{c}\text { Grupo BT } \\
\text { N } 107 \\
\end{array}$ & $\begin{array}{c}\text { Após uso } \\
\text { BT }\end{array}$ & $\begin{array}{c}\text { Após ART } \\
\text { BT } \\
\end{array}$ & $\begin{array}{c}\text { Grupo CTR } \\
\text { N } 105 \\
\end{array}$ & Após ART \\
\hline Idade & $\begin{array}{c}30,1(3,6 \%) \\
(27 \text { meses) }\end{array}$ & - & - & $\begin{array}{c}29,2(3,4 \%) \\
(26 \text { meses })\end{array}$ & - \\
\hline \multicolumn{6}{|l|}{ Sexo } \\
\hline$F$ & $61(57 \%)$ & - & - & $61(58,1 \%)$ & - \\
\hline M & $46(43,0 \%)$ & - & - & $44(41,9 \%)$ & - \\
\hline \multicolumn{6}{|l|}{ Renda } \\
\hline$<1 \mathrm{SM}$ & $10(9,4 \%)$ & - & - & $19(18,1 \%)$ & - \\
\hline $1 \mathrm{SM}$ & $55(51,4 \%)$ & - & - & $47(44,8 \%)$ & - \\
\hline 2 a 3 SM & $42(39,3 \%)$ & - & - & $39(37,1 \%)$ & - \\
\hline \multicolumn{6}{|c|}{ Medidas iniciais } \\
\hline \multicolumn{6}{|c|}{ VPT } \\
\hline Alegre & $11(10,3 \%)$ & $57(53,3 \%)$ & $78(72,9 \%)$ & $13(12,4 \%)$ & $2(1,9 \%)$ \\
\hline Neutro & $21(19,6 \%)$ & $37(34,6 \%)$ & $24(22,4 \%)$ & $19(18,1 \%)$ & $9(8,6 \%)$ \\
\hline Medo & $21(19,6 \%)$ & $8(7,5 \%)$ & $0(0,0 \%)$ & $18(17,1 \%)$ & $51(48,6 \%)$ \\
\hline Chorando & $13((12,1 \%)$ & $0(0,0 \%)$ & $3(2,8 \%)$ & $10(9,5 \%)$ & $7(6,7 \%)$ \\
\hline Triste & $21(19,6 \%)$ & $0(0.0 \%)$ & $1(0,9 \%)$ & $26(24,8 \%)$ & $24(22,9 \%)$ \\
\hline Pânico & $9(8,4 \%)$ & $5(4,7 \%)$ & $1(0,9 \%)$ & $7(6,7 \%)$ & $4(3,8 \%)$ \\
\hline \multicolumn{6}{|l|}{ FIS } \\
\hline Feliz & $5(4,7 \%)$ & $71(66,4 \%)$ & $104(97,2 \%)$ & $10(9,5 \%)$ & $2(1,9 \%)$ \\
\hline Assustado & $41(38,3 \%)$ & $30(28,0 \%)$ & $2(1,9 \%)$ & $29(27,6 \%)$ & $51(48,6 \%)$ \\
\hline Triste & $28(26,2 \%)$ & $1(0,9 \%)$ & $1(0,9 \%)$ & $33(31,4 \%)$ & $30(28,6 \%)$ \\
\hline Zangado & $17(15,9 \%)$ & $4(3,7 \%)$ & $090,0 \%)$ & $11(10,5 \%)$ & $9(8,6 \%)$ \\
\hline Chorando & $16(15,0 \%)$ & $1(0,0 \%)$ & $0(0,0 \%)$ & $22(21,0 \%)$ & $13(12,4 \%)$ \\
\hline \multicolumn{6}{|c|}{ FIS Turma da Monica } \\
\hline Muito feliz & $1(0,9 \%)$ & $37(34,6 \%)$ & $79(73,8 \%)$ & $2(1,9 \%)$ & $0(0,0 \%)$ \\
\hline Feliz & $8(7,5 \%)$ & $66(61,7 \%)$ & $26(24,3 \%)$ & $18(17,1 \%)$ & $2(1,9 \%)$ \\
\hline Assustado & $23(21,5 \%)$ & $2(1,9 \%)$ & $1(0,9 \%)$ & $23(21,9 \%)$ & $23(21,9 \%)$ \\
\hline Triste & $30(28,0 \%)$ & $2(1,9 \%)$ & $1(0,9 \%)$ & $24(22,9 \%)$ & $38(36,2 \%)$ \\
\hline Chorando & $45(42,1 \%)$ & $0(0,0 \%)$ & $0(0,0 \%)$ & $38(36,2 \%)$ & $42(40,0 \%)$ \\
\hline
\end{tabular}

Legenda: $\mathrm{BT}=$ Brinquedo terapêutico, $\mathrm{ART}=$ Tratamento restaurador Atraumático, VPT $=$ Venham Picture Test, FIS= Facial Image Scale.

Fonte: Carvalho MTF, et al., 2019. 
Na Figura 2 os dados das medidas de ansiedade na FIS Turma da Mônica são apresentados no diagrama do tipo aluvial, nos dois momentos da intervenção. O conjunto de observações são representados por um fluxo demonstrando as mudanças das frequências para cada nível. A altura da barra representa as crianças em estudo. Simultaneamente as faixas cinza (BT) e laranja (CTR) fornecem uma ideia do "fluxo". Assim, temos representado os grupos BT e CTR onde pode ser observado as mudanças de níveis de ansiedade na escala FIS T da Mônica ("MUITO FELIZ", "FELIZ", "ASSUSTADO", "TRISTE" e "CHORANDO") antes e após ART.

Apresenta uma tendência de redução da ansiedade avaliada pelo instrumento do FIS Turma da Mônica com frequências indicativas de maior ansiedade antes do BT e no grupo CTR, onde, após o uso do BT é observado a mudança de níveis de "FELIZ", "ASSUSTADO", "TRISTE" e "CHORANDO" para os níveis de "MUITO FELIZ" e "FELIZ", após a ART. O grupo CTR, onde não foi aplicado o BT, após o procedimento não invasivo ART, os níveis de ansiedade aumentaram. Mesmas características são observadas com o diagrama aluvial das medidas de ansiedade VPT e FIS, nos dois momentos da intervenção, antes e após ART, em ambos os grupos.

Figura 2 - Diagrama aluvial das medidas de ansiedade FIS Turma da Mônica aplicada em todas as crianças da pesquisa.

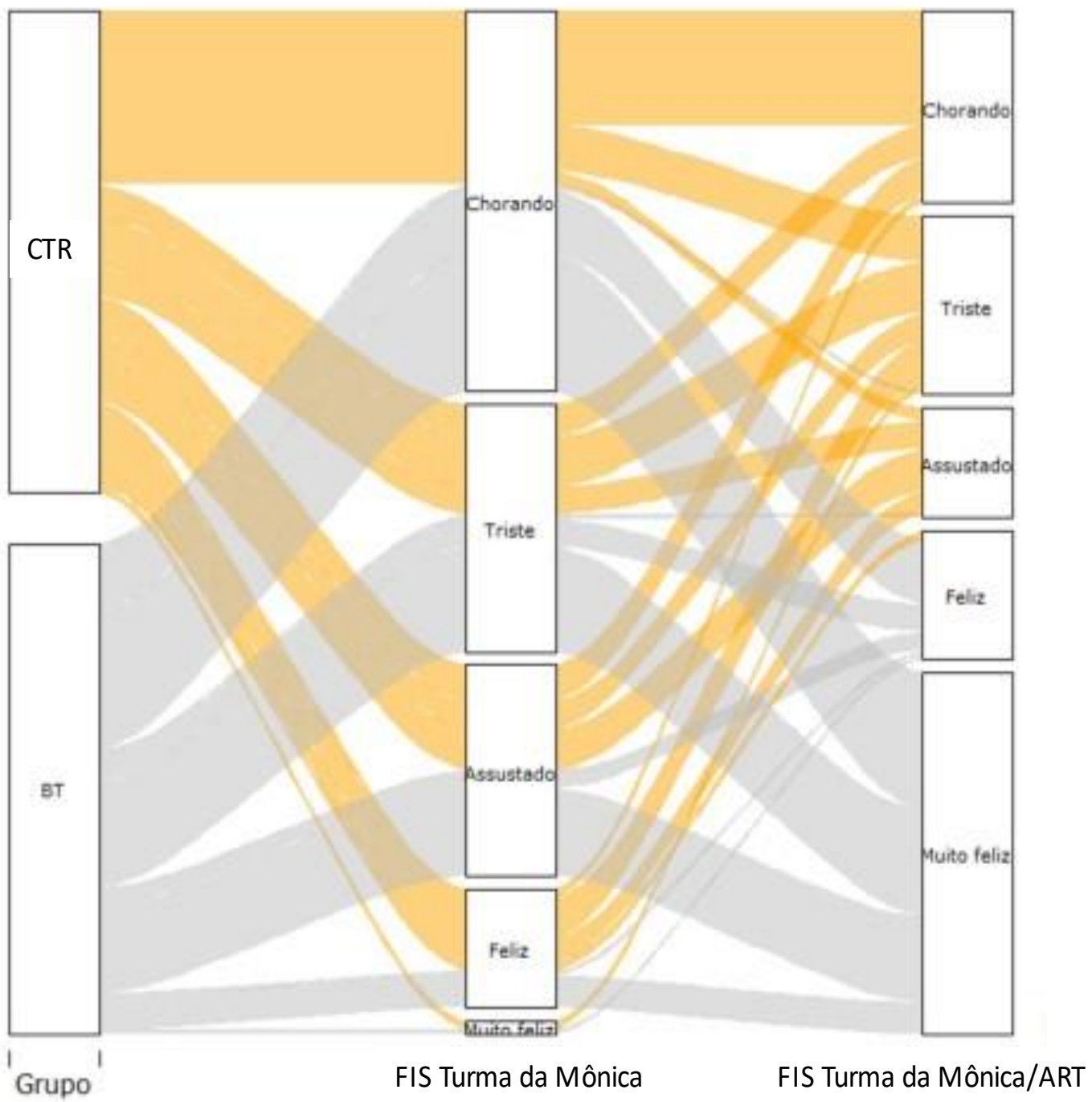

Legenda: CTR= Controle; BT= Brinquedo; FIS=Facial Image Scale; ART= Tratamento restaurador Atraumático.

Fonte: Carvalho MTF, et al., 2019. 
Na Figura 3 temos a representação gráfica referente ao parâmetro biológico, frequência cardíaca (batimentos cardíacos/bpm) de todas as crianças do estudo (grupo BT e grupo CTR) onde, cada ponto da amostra é codificada conforme o grupo, laranja para CTR e cinza para BT. Pode-se observar que após uso do brinquedo a faixa de pulsação das crianças do grupo BT reduziu-se de 110 a 124 bpm da medida inicial para 99 a 115 após o BT. Na fase pós ART, a faixa de pulsação vai de 103 a 118 bpm, assim, menor que a inicial.

Figura 3 - Boxplots da pulsação de todas as crianças do estudo. Adicionalmente cada ponto da amostra, codificada conforme o grupo do estudo, laranja para controle e cinza para BT, estão sobrepostos aos diagramas, mostrando a totalidade de dados.

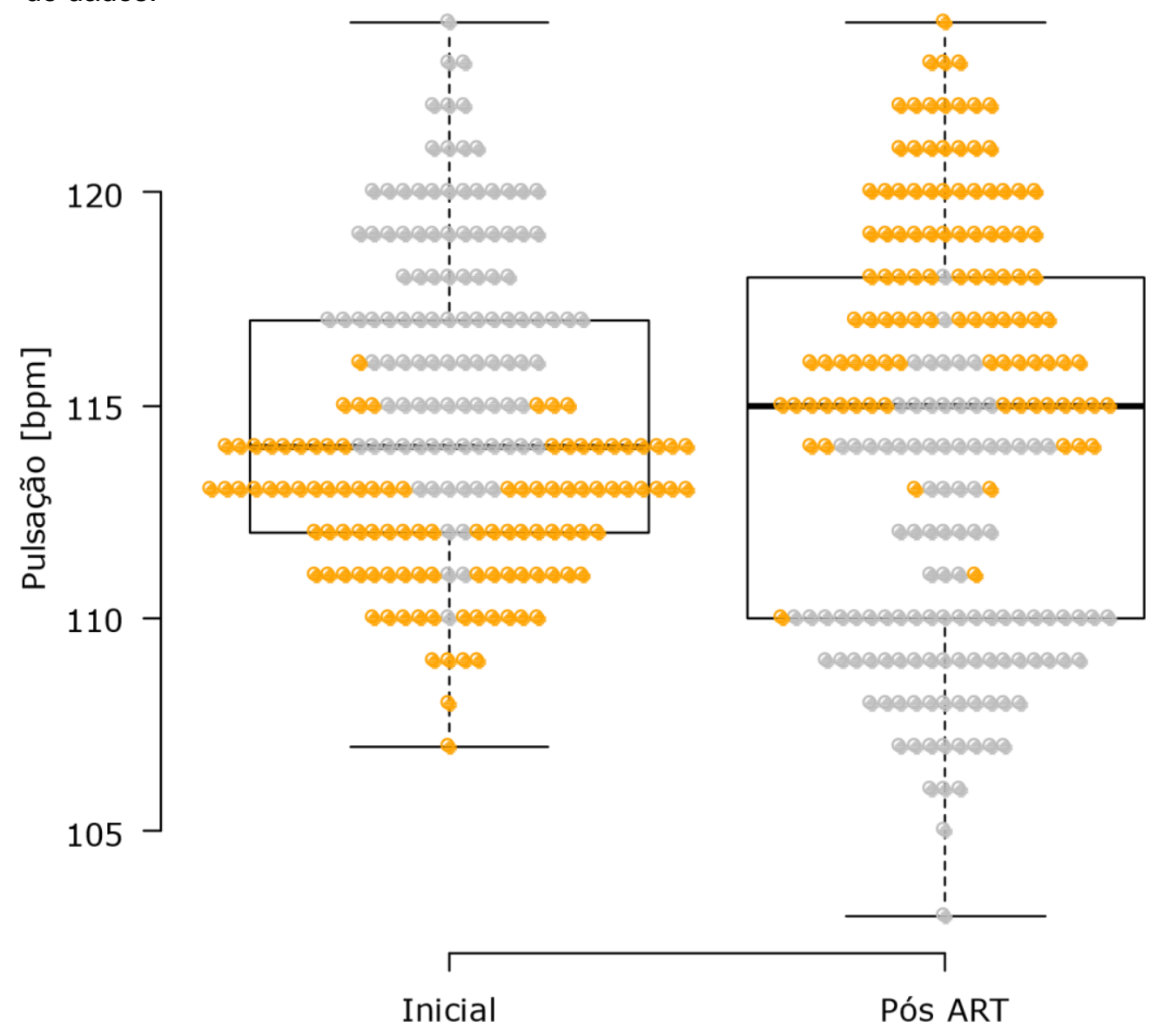

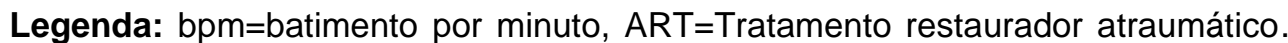

Fonte: Carvalho MTF, et al., 2019.

Para comparações das medidas de ansiedade empregou-se teste Mann Whitney para grupos independentes. Figura 4 mostra frequências por nível de ansiedade medidas após ART. Barras cinzas, grupo BT e laranjas, grupo CTR. Observa-se ansiedade após ART pelo VPT de posto inferior ao do grupo BT, figura "ALEGRE" (ausência de ansiedade). Uso da FIS após ART também apresentou ansiedade de posto inferior ao do grupo BT, carinha "FELIZ". A ansiedade também foi apontada de posto inferior após ART pelo FIS Turma da Mônica no grupo BT, carinha "MUITO FELIZ".

As crianças do Grupo CTR selecionaram as figuras "MEDO", seguido de "TRISTE", refletindo ansiedade (VPT). O grupo CTR, que não utilizaram o BT para trabalhar a ansiedade, apontaram carinha "ASSUSTADO" seguido da "TRISTE"; demonstrando a ansiedade presente (FIS). Já a leitura das barras laranjas (grupo CTR) apresentaram resultados pela preferência das carinhas "CHORANDO", "TRISTE" e "ASSUSTADO" (FIS Turma da Mônica) demonstrando, também, grau de ansiedade presente de acordo com o escore da escala. 
Figura 4 - Gráfico de barras adjacentes para o VPT após o ART; FIS adaptado após ART e FIS Turma da Mônica após ART para todas as crianças do estudo.

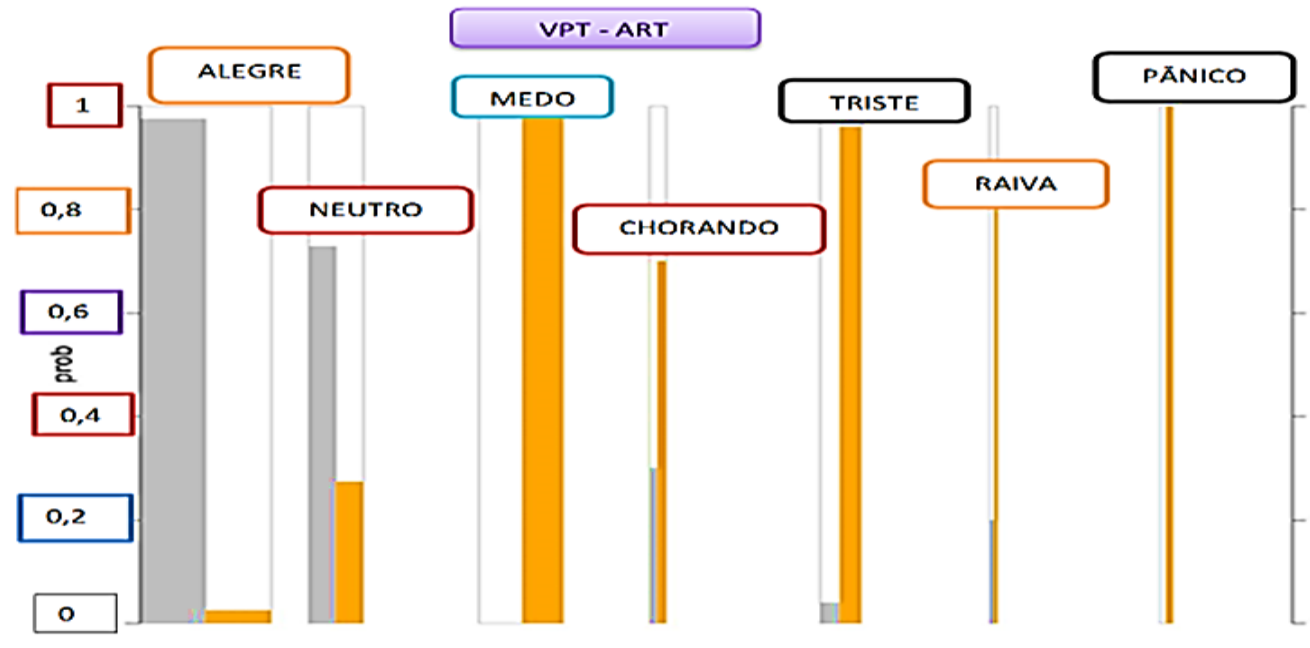

FIS APOS ART

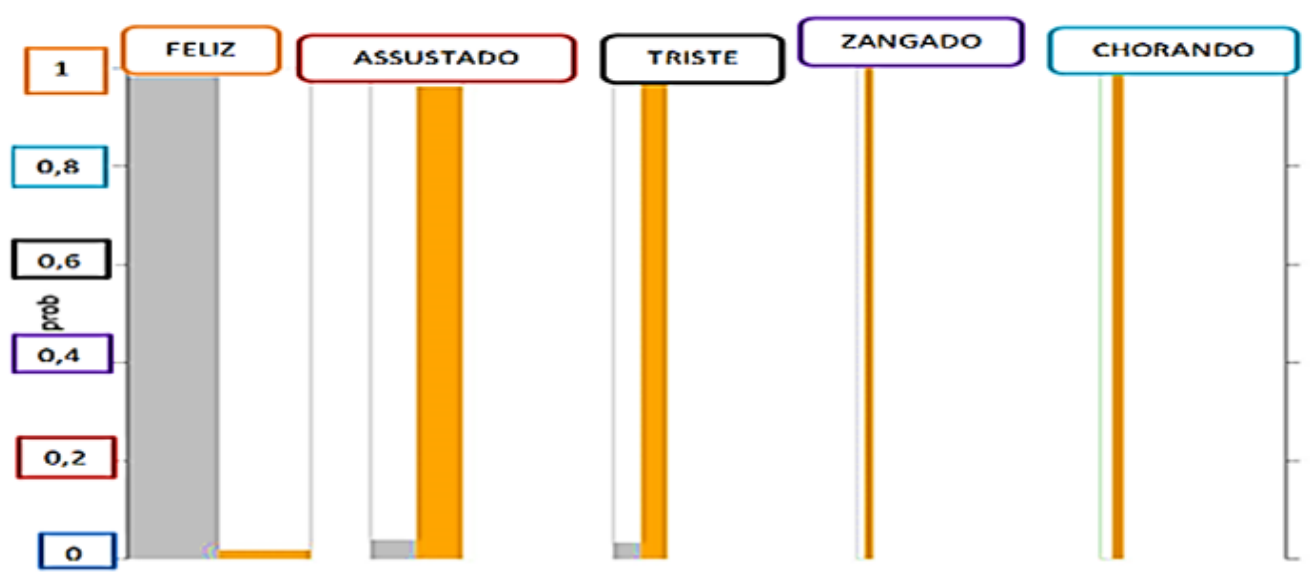

FIS T DA MONICA APOS ART

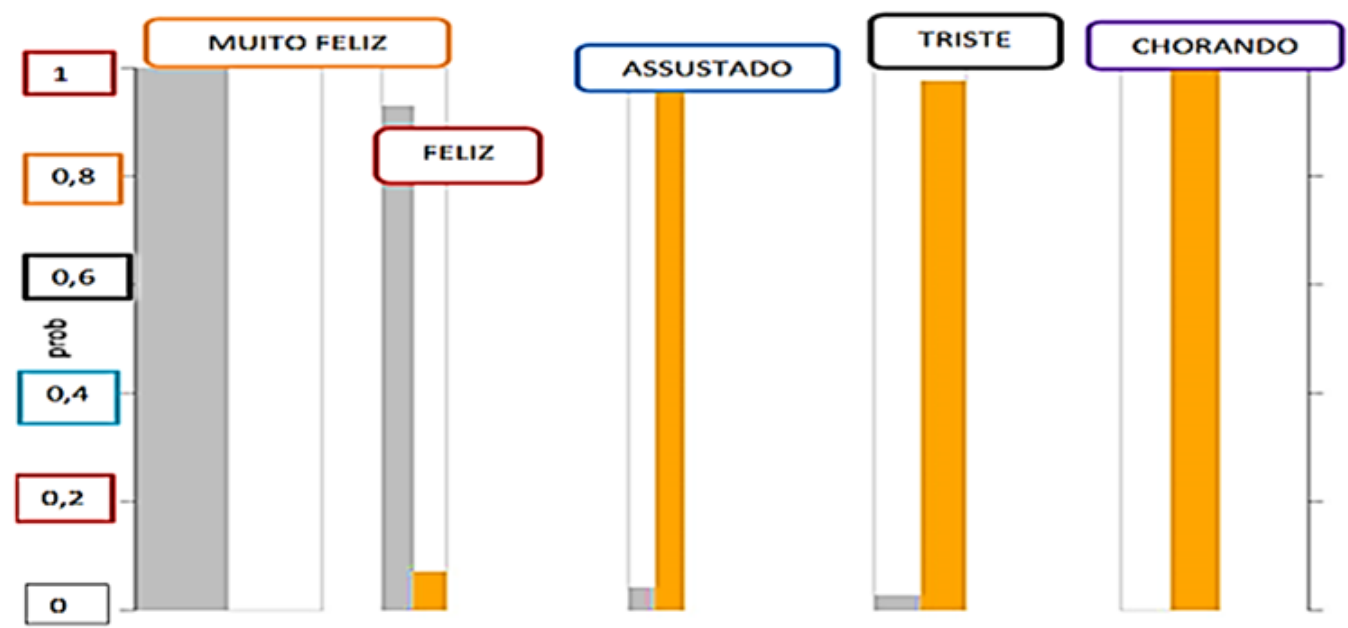

Legenda: VPT = Venham Picture Test; ART=Tratamento restaurador Atraumático, FIS= Facial Image Scale, ${ }^{*} \mathrm{VPT}$ barras (da esquerda para direita): Alegre-Neutro-MedoChorando-Triste-Raiva-Pânico; ${ }^{* * *}$ FIS adaptado: Feliz-Assustado-Triste-ZangadoChorando; ${ }^{* * * *}$ FIS Turma da Mônica: Muito Feliz-Feliz-Triste-Assustado-TristeChorando. Fonte: Carvalho MTF, et al., 2019. 
A Figura 5 apresenta as matrizes de diagramas de dispersão para as variáveis das medidas de pulsação, VPT, FIS e FIS Turma da Mônica para as medidas iniciais, após o BT (apenas para o grupo BT) e após o ART para todas as crianças do estudo. As diagonais das matrizes contêm histogramas de cada variável identificada. As células da parte inferior da matriz contêm os valores das correlações de Spearman e no canto superior direito dessas células encontra-se o código de significância estatística dessas correlações, cuja legenda encontra logo abaixo da figura. As categorias ordinais são plotadas pelos numerais correspondentes aos postos dessas escalas, em vez dos nomes dos níveis delas e que cada blotagem dos pontos refere-se ao valor que cada criança possui. Quanto mais alto o valor maior é a correlação. Essa correlação foi constatada entre o teste (VPT), as escalas de ansiedade (FIS e FIS Turma da Mônica) e a frequência cardíaca (bpm) onde foi obtido $\mathrm{p}=0,001$.

Figura 5 - Matriz de diagramas de dispersão para as medidas após o ART para todas as crianças do estudo.

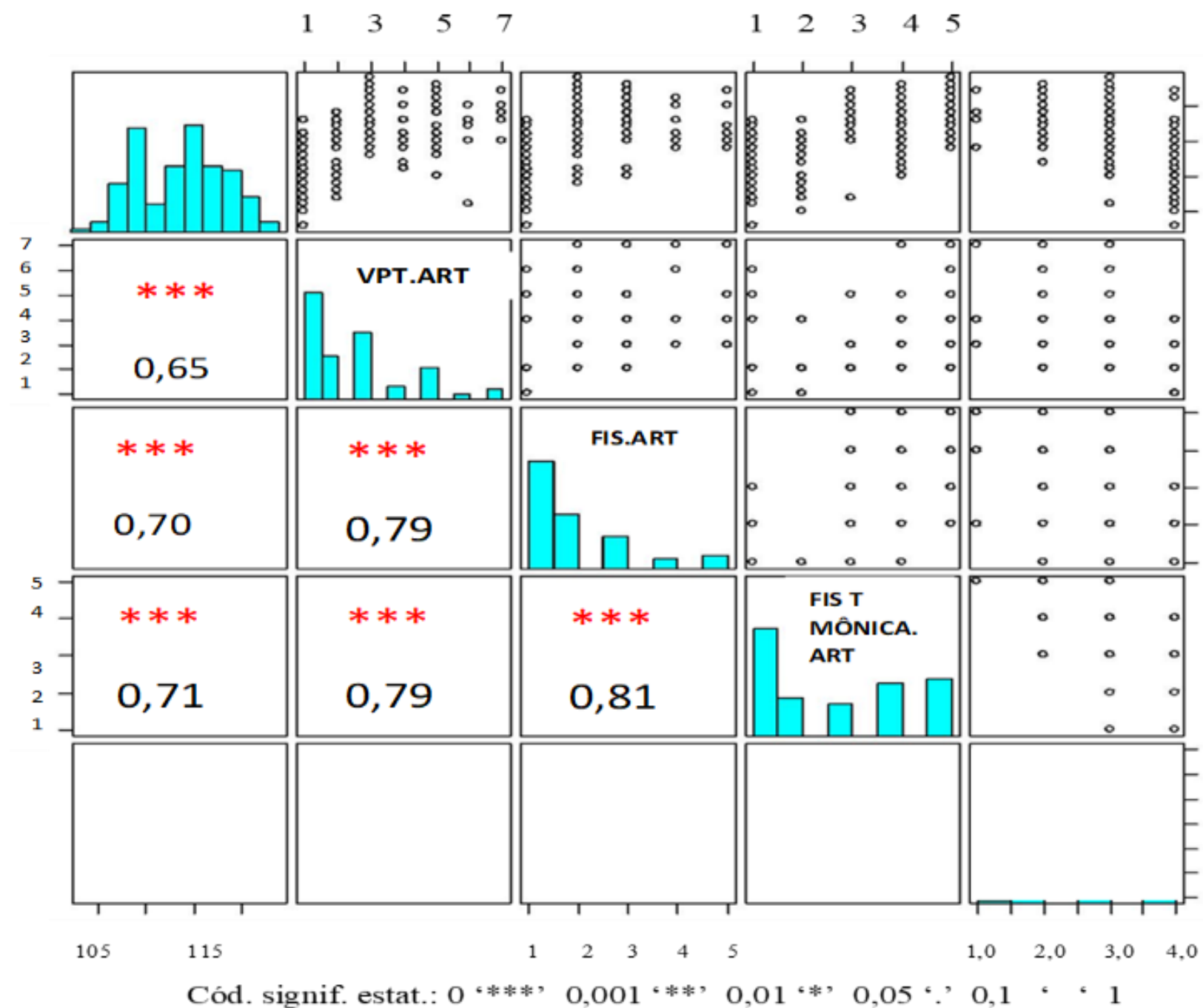

Legenda: VPT=Venham Picture Test; FIS=Facial Image Test; ART= Tratamento Restaurador Atraumático, Fonte: Carvalho MTF, et al, 2019.

\section{DISCUSSÃO}

O presente estudo fundamenta a relevância da utilização do BT na assistência à criança, quando submetida a procedimentos odontológicos, demonstrando que essa estratégia reduz o grau de ansiedade. Os resultados mostraram que o grupo de crianças que utilizaram o BT exibiram menor grau de ansiedade e uma frequência cardíaca mais baixa quando comparado ao grupo de crianças que não experimentaram o BT. Da 
mesma forma é notável a redução da ansiedade quando avaliados sob a óptica das escalas faciais e do teste VPT. Os dados tornam-se ainda mais relevantes, quando consideramos a faixa etária estudada, haja vista que além de pouco estudada sob o ponto de vista de comportamento, configura um desafio no atendimento clínico para o cirurgião dentista, bem como para o núcleo familiar (MOURA LA, et al., 2016; OLIVEIRA MF, et al., 2012).

Pesquisas demonstram que crianças de menor idade são mais ansiosas e essa ansiedade decresce à medida que avançam em suas faixas etárias (ARAÚJO IS, et al., 2018; RIBEIRO IR, 2016). Tal fato não foi observado em nossos dados, provavelmente em função das diferenças de idade da população estudada serem pequenas.

Relativo ao sexo, não houve associação entre ansiedade, parâmetros biológicos e escalas faciais, o que se ajusta aos resultados de (OLIVEIRA HBS e FUMIS RRL, 2018; FERREIRA HACM e OLIVEIRA AMG, 2017; CARACIOLO GM, 2008; RIBAS TA, et al., 2006). Entretanto, Bottan E, et al. (2008), Martins N e Dias MR (2016) apontam o sexo feminino com maior ansiedade, sugerindo que meninas tendem a ter maior facilidade para expor suas emoções.

A frequência cardíaca é um importante marcador biológico do nível de ansiedade e do medo (TOPALOGLU AK, et al., 2007). Em nosso estudo, essa medida foi acentuada no grupo BT, em comparação ao CTR, previamente ao tratamento. Pode-se creditar esses valores ao fato de que à apresentação do BT às crianças potencializa suas emoções no sentido de estimulá-la a brincar, aumentando momentaneamente o nível de ansiedade (NAGANO HCM, 2010; FERREIRA HACM e OLIVEIRA HMG, 2017).

Sob a ação de situações desconhecidas, crianças tendem a alterar a linha de base da frequência respiratória e como consequência aumentar o índice de oxigenação e frequência cardíaca. Entretanto, no grupo BT, antes e após o uso do BT e do procedimento odontológico e no grupo CTR, antes e após ART, a média de saturação de oxigênio manteve-se semelhante, sem diferença estatisticamente significante $(p>0,05)$ (TSHISWAKA SK e PINHEIRO SL, 2020; PRADO IM, et al., 2019).

Há que se enfatizar que as crianças incluídas nesse estudo detinham alguma (até duas consultas) ou nenhuma vivência no ambiente odontológico. Essa circunstância associada ao procedimento odontológico, a princípio de baixo potencial para gerar ansiedade, ilustra a simetria destes dados. A ansiedade odontológica parece diminuir com o aumento da idade do indivíduo, o que pode ser explicado pela maior exposição da criança com a odontologia (FERREIRA HACM e OLIVEIRA HMG, 2017; GAMA TS, et al., 2017). Por outro lado, essa afirmação exige melhor avaliação, haja vista que pesquisas mostram igualmente um aumento na ansiedade a partir da segunda consulta em crianças de tenra idade (GÓES MPS, et al., 2010; MARTINS N e DIAS MR, 2016; MELO RB, et al., 2015).

Com intuito de verificarmos a correlação entre as três escalas de ansiedade e a frequência cardíaca, recorreu-se a análise de correlação de postos de Spearman. Verificou-se uma correlação positiva entre os escores VPT, FIS, FIS T da Mônica e frequência cardíaca ( $p=0,001)$. Essa correlação também foi encontrada no teste de correlação entre as duas escalas VPT e FIS. (FRENCKEN JE, 2017). Folayan MO, et al. (2004) afirmam que a cultura influencia o contexto da experiência de ansiedade, assim como a interpretação do seu significado e a sua responsividade.

O valor do brincar foi destacado por Maluf ÂCM (2014), descrevendo seus benefícios, entre eles, a utilização do BT na promoção de uma relação terapêutica (MELO RB, et al., 2015; BARROSO MCCS, et al., 2020). O BT pode ser de três tipos: dramático, instrucional e capacitador (CALEFFI CCF, et al., 2016). No atual estudo, foi utilizado o brinquedo terapêutico instrucional (Play Doh Doctor), onde a partir dele, as crianças reproduziram o procedimento a ser realizado posteriormente nelas. Segundo Pontes JED, et al. (2015), Melo L, et al. (2008) crianças preparadas com o BT se sentem mais tranquilas e colaborativas, e superam mais facilmente as adversidades inerentes aos procedimentos. Acredita-se que o brincar é o primeiro experimentar do mundo que se realiza na vida da criança (MORAES VM, 2020).

A utilização do BT, nesse estudo, foi avaliada através das escalas de ansiedade VPT, FIS e FIS (Turma da Monica). A avaliação da reação do paciente infantil, baseada nestas escalas, é oportuna, permitindo 
manifestar sua sensação ao apontar a figura que compõe as escalas, reduzindo a necessidade de expressar verbalmente, especialmente crianças de pouca idade, como a estudada nessa pesquisa (GIBILINI C, et al., 2011; SILVA RDM, et al., 2017).

Nossos resultados demonstraram que as crianças que utilizaram o BT tiveram uma relação positiva com o procedimento odontológico, menor ansiedade, frequência cardíaca mais baixa em relação aos participantes do grupo CTR. Tais resultados, se assemelham aos de Ribeiro CA (2008), que afirma que o BT antecipa a realidade a ser vivenciada pela criança, minimizando o medo e a ansiedade, construindo vínculo entre a criança e o profissional (RIBEIRO WA, et al., 2020). Por outro lado, estudos identificam algumas dificuldades na utilização do BT, pela própria resistência do profissional a novas propostas e protocolos de trabalho (GAMA TS, et al., 2017; SILVA RDM, et al., 2017; RIBEIRO WA, et al., 2020).

Relativo ao delineamento do estudo, destacamos os seguintes limites: faixa etária das crianças avaliadas e o baixo potencial do procedimento eleito para produzir ansiedade. A despeito das técnicas e escalas projetivas serem indicadas para crianças jovens, possivelmente, uma maior habilidade cognitiva, em função da idade, qualificaria as respostas. Todavia essas limitações foram compensadas pelos cuidados metodológicos na coleta de dados e na condução da intervenção clínica.

Os resultados da pesquisa realçam que o desempenho profissional não deve limitar-se aos conhecimentos científicos e habilidades técnicas, mas agregar a eles a competência de incluir um recurso terapêutico humanizado e que envolva aspectos biopsicossociais. O acolhimento do profissional da saúde confere à criança e seu núcleo familiar o status de sujeito e não de objeto. Essa condição, seguramente, reduz a ansiedade da criança e aumenta sua habilidade no enfrentamento do procedimento clínico e de outras adversidades. O BT integra a equipe de saúde, criança e família, promovendo a humanização nesse cuidado do indivíduo.

Nessa pesquisa o BT se fortalece como ferramenta para abordagem humanizada e propícia nas intervenções de rotina odontopediátrica promovendo: comportamento colaborativo da criança; estreita a comunicação entre o cirurgião dentista e a criança; atua como facilitador da compreensão do universo da criança. A aplicação do BT na prática clínica deve ser estimulada, considerando que sua utilização promove um alívio na ansiedade, atenua as reações negativas e auxilia na adesão ao tratamento por parte das crianças.

\section{CONCLUSÃO}

O Brinquedo Terapêutico e o ato de brincar demonstrou ser uma estratégia que atenua as adversidades de um tratamento odontológico. Essa prática está intimamente vinculada à humanização do cuidado, respeitando os valores da criança e de seu núcleo familiar. A aplicação do BT promoveu redução do nível de ansiedade, tornando as crianças mais receptivas ao procedimento odontológico. Revelou-se, ainda, um método positivo e apropriado para estimular seu uso na rotina clínica odontopediátrica.

\section{REFERÊNCIAS}

1. ARAÚJO IS, et al. Avaliação do nível de ansiedade prévio à consulta odontológica em crianças de 5 a 12 anos. Revista Interfaces: saúde, humanas e tecnologia, 2018; 6(16): 59-67.

2. BARROSO MCCS, et al. Percepção das crianças acerca da punção venosa por meio do brinquedo terapêutico. Acta Paulista de Enfermagem, 2020; 33.

3. BOTTAN E, et al. Ansiedade ao tratamento odontológico: estudo exploratório com crianças e adolescentes de um município de Santa Catarina. Revista Sul-Brasileira de Odontologia. 2008; 5(1): 13-19.

4. CALEFFI CCF, et al. Contribuição do brinquedo terapêutico estruturado em um modelo de cuidado de enfermagem para crianças hospitalizadas. Revista Gaúcha Enfermagem. 2016; 37(2), 58131.

5. CARACIOLO GM. Medo e/ou ansiedade como fator inibitório para visita ao dentista em pré-escolares. Tese. (Doutorado em Odontologia). Faculdade de Odontologia da Universidade de Pernambuco, Camaragibe, PE; 2008; $156 \mathrm{p}$.

6. CARVALHO FS, et al. Assistência do Enfermeiro à criança no tratamento oncológico junto ao Brinquedo Terapêutico. Revista Cuidados em Saúde, 2015; 9(3): 1-11. 
7. CÉ L. Fatores de influência e de predição da ansiedade e comportamento do paciente odontopediátrico: métodos para à avaliação da ansiedade. Monografia (Especialização em Odontopediatria). Universidade Federal de Santa Catarina, Florianópolis, SC, 2002. 166 p.

8. CLARO MT. Escala de faces para avaliação da dor em crianças: etapa preliminar. Dissertação (Mestrado em Enfermagem) - Escola de Enfermagem. Universidade de São Paulo, Ribeirão Preto, 1993. 50 p.

9. DIMAIRO M, et al. The adaptive designs CONSORT extension (ACE) statement: a checklist with explanation and elaboration guideline for reporting randomised trials that use an adaptive design. Trials. 2020; 21: 528.

10. FERREIRA HACM e OLIVEIRA AMG. Ansiedade entre crianças e seus responsáveis perante o atendimento odontológico. Revista de Odontologia da Universidade Cidade de São Paulo, 2017; 29(1): 6-17.

11. FRENCKEN JE. Atraumatic restorative treatment and minimal intervention. British Dental Journal, 2017 ; $22(3)$ : 183189.

12. FOLAYAN MO e IDEHEN EE. The modulating effect of culture on the expression of dental anxiety in children: a literature review. Int J Pediatr Dent. 2004 Jul; 14 (4): 241-5

13. GAMA TS, et al. Perfil do medo apresentado por crianças frente ao tratamento odontológico. Revista Uningá Review, 2017; 29(3): 23-27.

14. GHADIMI S, et al. Effect of visual distraction on children's anxiety during dental treatment: a crossover randomized clinical trial. European Archives of Paediatric Dentistry, 2018. v. 19, n. 4, p. 239-244

15. GIBILINI C, et al. Avaliação da autopercepção do tratamento restaurador atraumático pelo paciente infantil. Revista da Faculdade de Odontologia da Universidade de Passo Fundo, 2011; 16(1): 18-24.

16. GÓES MPS, et al. Ansiedade, medo e sinais vitais dos pacientes infantis. Odontologia Clínico Científica, 2010; 9(1): 39-44.

17. MALUF ÂCM. Brinquedoteca: um espaço estruturado para brincar. 4a ed. São Paulo: Ática, 2014.

18. MARTINS N, DIAS MR. Contágio emocional de ansiedade encarregado de educação/crianças em odontopediatria. Revista Portuguesa de Estomatologia, Medicina Dentária e Cirurgia Maxilofacial, 2016; 57(3): 164-170.

19. MARWAH N, et al. Music distraction - its efficacy in management of anxious pediatric dental patients. Journal of Indian Society of Pedodontics and Preventive Dentistry, 2005; 23(4): 168-170.

20. MELO RB, et al. Avaliação da relação entre procedimentos odontológicos e comportamento infantil. Revista Odontlógica do Brasil Central, 2015; 24(68): 20-5.

21. MELO L, et al. O brinquedo terapêutico como facilitador na adesão ao tratamento de diabetes mellitus tipo 1 na infância. Pediatr. mod, 2008. p. 100-103.

22. MORAES VM. O 'brincar' na educação infantil e o desenvolvimento da criança. Educationis, 2020; 8(1): 1-7.

23. MOURA LA, et al. Prevalência e fatores associados à ansiedade pré-operatória em crianças de 5 a 12 anos. Revista Latino-Americana de Enfermagem, 2016; 24: e. 2708.

24. NAGANO HCM. Dilemas e reflexões de odontopediatras sobre estratégias de manejo do comportamento infantil. Tese (Doutorado em Odontopediatria). Faculdade de Odontologia da Universidade Federal de Santa Catarina, Florianópolis, SC, 2010; $93 \mathrm{p}$.

25. OZKALAYCI O, et al. Effects of music on sedation depth and sedative use during pediatric dental procedures. Journal of Clinical Anesthesia. 2016; 34: 647-653.

26. OLIVEIRA HSB, FUMIS RRL. Influência do sexo e condição de cônjuge nos sintomas de ansiedade, depressão e transtorno de estresse pós-traumático em pacientes admitidos à unidade de terapia intensiva e em seus respectivos cônjuges. Revista Brasileira de Terapia Intensiva, 2018; 30(1): 35-41.

27. OLIVEIRA MF, et al. Avaliação da ansiedade dos pais e crianças frente ao tratamento odontológico. Pesquisa Brasileira em Odontopediatria e Clínica Integrada, 2012; 12(4): 483-489.

28. PONTES JED, et al. Brinquedo terapêutico: preparando a criança para a vacina. Einstein, 2015; 13(2): $238-242$.

29. PRADO IM, et al. Use of distraction techniques for the management of anxiety and fear in paediatric dental practice: A review of randomized controlled trials. International Journal of Paediatric Dentistry, 2019; 29(5): 650-668.

30. RANK RCIC, SILVA DRP. Avaliação das reações emocionais em crianças com idade entre 2 a 8 anos, frente a uma sala de espera infantil. FOA, 2002; 4(2): 47-53.

31. RIBAS TA, et al. Avaliação da ansiedade odontológica de crianças submetidas ao tratamento odontológico. Arquivos em Odontologia, 2006; 42(3):190-198.

32. RIBEIRO CA, et al. A criança e o brinquedo no hospital. Almeida FA, Sabatés AL, organizadoras. Enfermagem pediátrica: a criança, o adolescente e sua família no hospital. Barueri: Manole, 2008; 65-77.

33. RIBEIRO IR. Avaliação da ansiedade infantil frente ao tratamento odontológico através da escala visual analógica (EVA). Revista Fluminense de Odontologia, 2016; 1(45):1-17.

34. RIBEIRO WA, et al. Contributos do brinquedo terapêutico no processo de cuidado a criança hospitalizada: um estudo da literatura. Research, Society and Development, 2020; 9(7): e1000974706-e.

35. SOARES JP, et al. Avaliação do comportamento odontológico infantil durante procedimentos de profilaxia e exodontia. Arquivos em Odontologia, 2020; 56: e04.

36. SILVA RDM, et al. Brinquedo terapêutico no preparo de crianças para procedimentos invasivos: Revisão Sistemática. Jornal de Pediatria, 2017; 93(2): 6-16.

37. TOPALOGLU AK, et al. Perceived dental anxiety among schoolchildren treated through three caries removal approaches. Journal of Applied Oral Science, 2007; 15(3): 235-240.

38. TSHISWAKA SK, PINHEIRO SL. Effect of music on reducing anxietyin children during dental treatment. Revista Gaúcha de Odontologia, 2020; 68: e20200033.

39. VENHAM LL, et al. Interval rating scales for children's dental anxiety and uncooperative behavior. Pediatric Dentistry, 1980; 2(3): 195-202.

40. ZHU M, et al. Experiential learning for children's dental anxiety: a cluster randomized trial. BioMed Central Oral Health, 2020; 31(20): 216-219. 